\title{
Vacancy cluster growth and thermal recovery in hydrogen-irradiated tungsten
}

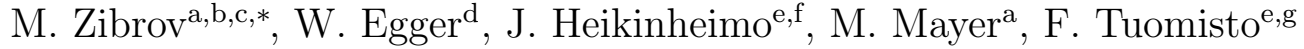 \\ ${ }^{a}$ Max Planck Institute for Plasma Physics, 85748 Garching, Germany \\ ${ }^{b}$ Physik-Department E28, Technische Universität München, 85748 Garching, Germany \\ ${ }^{c}$ Department of Applied Physics, Ghent University, 9000 Ghent, Belgium \\ ${ }^{d}$ Institut für Angewandte Physik und Messtechnik, Universität der Bundeswehr München, \\ 85577 Neubiberg, Germany \\ ${ }^{e}$ Department of Applied Physics, Aalto University, P.O. Box 15100 FI-00076 Aalto, \\ Finland \\ ${ }^{f}$ VTT Technical Research Centre of Finland Ltd, Nuclear Safety, P.O. Box 1000 \\ FI-02044 VTT, Finland \\ ${ }^{g}$ Department of Physics and Helsinki Institute of Physics, University of Helsinki, P.O. \\ Box 43, FI-00014 Helsinki, Finland
}

\begin{abstract}
The thermal evolution of vacancies and vacancy clusters in tungsten (W) has been studied. W (100) single crystals were irradiated with $200 \mathrm{keV}$ hydrogen $(\mathrm{H})$ ions to a low damage level $\left(5.8 \times 10^{-3} \mathrm{dpa}\right)$ at $290 \mathrm{~K}$ and then annealed at temperatures in the range of $500-1800 \mathrm{~K}$. The resulting defects were characterized by positron annihilation lifetime spectroscopy (PALS) and positron annihilation Doppler broadening spectroscopy (DBS). Annealing at $700 \mathrm{~K}$ resulted in the formation of clusters containing 10-15 vacancies, while at $800 \mathrm{~K}$ and higher temperatures clusters containing about 20 vacancies or more were formed. Reduction of the defect concentration likely accompanied by further coarsening of the clusters started at $1300 \mathrm{~K}$ and ended at $1800 \mathrm{~K}$ with the complete defect recovery. The determined cluster sizes at $700 \mathrm{~K}$ and $800 \mathrm{~K}$ were larger than the estimated minimum cluster sizes that are thermally stable at these temperatures, indicating that the migration and ensuing coalescence of small clusters plays an important role in cluster growth.
\end{abstract}

\footnotetext{
${ }^{*}$ Corresponding author

Email address: Mikhail.Zibrov@ipp.mpg.de (M. Zibrov)
} 
Keywords: Tungsten, Radiation defects, Vacancies, Vacancy clusters, Positron annihilation

\section{Introduction}

One of the key issues on the way of the development of fusion power plants is the selection of proper plasma-facing materials (PFMs) [1]. Due to its high melting temperature, high thermal conductivity, high sputtering threshold, and relatively low long-term activation after neutron irradiation, tungsten $(\mathrm{W})$ is nowadays considered as one of the most promising materials [2, 3]. It was chosen as the divertor material for the international experimental fusion reactor ITER, and is also considered as the divertor and the first wall material for future fusion devices, such as a demonstration fusion reactor (DEMO). In fusion reactors with a "burning" deuterium-tritium plasma, PFMs are subjected to intense fluxes of $14 \mathrm{MeV}$ neutrons that introduce radiation defects (vacancies and interstitial atoms, vacancy clusters, dislocation loops, etc.) through the whole material thickness. Neutrons also cause material transmutation, resulting in its activation and production of both hydrogen $(\mathrm{H})$ and helium (He). This alters the thermo-mechanical properties of the materials (degradation of thermal conductivity, embrittlement, swelling, and irradiation creep). Radiation defects serve as trapping sites for $\mathrm{H}$ isotopes, hence, their presence can significantly increase the tritium inventory in PFMs. These issues govern the possibility of a safe and economically feasible operation of a fusion power plant.

PFMs will be operated at elevated temperatures, resulting in a thermal evolution of the radiation defects [2, 3, 4, 5]. Vacancies and interstitials can migrate by thermally activated diffusion and annihilate either by recombining with each other or at extended sinks (free surfaces, grain boundaries, and dislocations). They can also agglomerate into more stable extended defects, such as vacancy clusters and dislocation loops. Vacancies, interstitials, and their clusters can also trap impurities (e.g., H, C), which may reduce their mobility and also prevent their annihilation. There are five major temperature regions of defect annealing (recovery) in metals [4, 5. They are linked either with the mobility of certain defect types or with the dissociation of defect clusters and defect-impurity complexes.

To unravel the mechanisms governing each recovery stage, post-irradiation isochronal annealing experiments are typically carried out. The summary of 
these studies for $\mathrm{W}$ can be found in [4, 6]. Although the reported temperature ranges of the various stages have some scatter, it should be stressed that these temperatures are not universal physical quantities since they depend on the used annealing scheme and on the concentration of defects, defect sinks, and impurities [4, 7]. The corresponding fundamental quantities are the activation energies for the respective processes. The annealing studies of neutron-irradiated $\mathrm{W}$ allowed the characteristic temperatures and activation energies of different stages to be identified, but the interpretation of the results is complicated due to the presence of several types of radiation defects in the samples. Basic studies of the annealing stages of the simplest type of radiation defect, Frenkel pairs (vacancies and interstitials) introduced by $\mathrm{MeV}$ electron irradiation, have also been carried out. However, most of them were performed by measuring the electrical resistivity recovery which is sensitive to the presence of defects, but the corresponding changes of the defect structure cannot be identified. Direct observations using transmission electron microscopy (TEM) are possible only for defect clusters larger than about $1 \mathrm{~nm}$.

Positron annihilation spectroscopy (PAS) is sensitive to open-volume defects with sizes ranging from single vacancies to TEM-visible cavities [8]. The existing positron annihilation Doppler broadening spectroscopy (DBS) studies of the recovery of (predominantly) Frenkel pairs in W introduced by light ion $(\mathrm{H}, \mathrm{He})$ irradiation to low damage levels covered a broad temperature range $(423-1900 \mathrm{~K})[7,9,10,11$. They were able to elucidate vacancy agglomeration in clusters during the recovery stage III, further coarsening of clusters at higher temperatures, and eventual recovery of the defects. However, the sizes of formed vacancy clusters could not be determined from DBS measurements. Positron annihilation lifetime spectroscopy (PALS) is capable of determining sizes of small vacancy clusters. However, in the studies by Sato et al. [12] and Heikinheimo et al. [13] the damage levels were too low to observe vacancy cluster formation. It has been detected by de Vries [14], but the measurements were carried only up to $900 \mathrm{~K}$. Ogorodnikova et al. [15] has also observed vacancy clustering, but the complete defect recovery took place already at $1173 \mathrm{~K}$, indicating that the defect concentration was not sufficiently high to observe the high-temperature recovery stage V previously observed by DBS [7, 9, 11].

This paper reports on the thermal evolution of vacancies and vacancy clusters in $\mathrm{W}$ in a wide range of temperatures (500-1800 K) investigated using both DBS and PALS techniques. Since the recovery stages I and II 
in $\mathrm{W}$ related with the mobility of self-interstitials and their complexes take place already below room temperature [4, 13], they are not relevant for fusion applications and are not examined in the present study. To minimise the impact of intrinsic defects in the material, such as impurities, dislocations, and grain boundaries, high-purity single crystalline $\mathrm{W}$ samples are used. Mostly Frenkel pairs are introduced as primary defects in the specimens via irradiation with $200 \mathrm{keV} \mathrm{H}$ ions to a low damage level $\left(5.8 \times 10^{-3} \mathrm{dpa}\right)$. In this case the damaged zone is about $1 \mu \mathrm{m}$ thick, which makes the surface a dominant and well-defined sink for the defects.

\section{Experimental details}

\subsection{Sample preparation}

The specimens were prepared from a high-purity single crystalline W rod grown along the $\langle 100\rangle$ direction by electron-beam floating zone melting at the Institute of Solid State Physics (Chernogolovka, Russia) [16, 17]. The stated accuracy of the rod orientation is within $2^{\circ}$. The dislocation density in the as-grown crystals is in the range of $10^{9}-10^{11} \mathrm{~m}^{-2}$ and most of the dislocations are arranged in walls, forming low-angle grain boundaries with a mean grain size around $500 \mu \mathrm{m}$ [16, 17]. The major stated impurities are (in $10^{-6}$ at. fr.): $\mathrm{C}(<15.3), \mathrm{N}(<7.9), \mathrm{O}(<5.8), \mathrm{Na}(<2.4), \mathrm{Si}(<2.0)$, $\mathrm{P}(<1.8)$, and $\mathrm{S}(<1.7)$. The samples with $(100)$ crystal surfaces were cut by spark erosion from the rod to $10 \times 10 \times 1 \mathrm{~mm}^{3}$. The residue from the cutting was removed by grinding of the samples with a set of $\mathrm{SiC}$ sandpapers with decreasing grit sizes (up to P4000). Then they were electrochemically polished to a mirror-like finish in a 1.5 wt. $\% \mathrm{NaOH}$ aqueous solution at a voltage of $19 \mathrm{~V}$. The samples were successively cleaned in an ultrasonic bath with acetone, isopropanol, and a high-purity acetone and then rinsed in deionized water. Analysis of two specimens after cutting and polishing by the Laue X-ray diffraction method showed that their surface normal is within $4^{\circ}$ of the $\langle 100\rangle$ direction. To reduce the possible spatial non-homogeneity of the microstructure and remove gaseous impurities, the specimens were first degassed at a temperature near $1350 \mathrm{~K}$ for $20 \mathrm{~min}$ and then annealed at about 2550-2600 K for $3 \mathrm{~min}$ (as a series of $30 \mathrm{~s}$ annealings) at a base pressure below $10^{-6} \mathrm{~Pa}$. 


\subsection{Ion irradiation}

In order to introduce mainly Frenkel pairs as primary defects, the samples were irradiated by $200 \mathrm{keV} \mathrm{H}$ ions in the implantation chamber connected to the $3 \mathrm{MV}$ tandem accelerator at IPP [18]. The residual pressure in the chamber is below $10^{-5} \mathrm{~Pa}$. The samples are clamped to a water-cooled copper $(\mathrm{Cu})$ holder by using molybdenum masks with an opening area of $8 \times 8 \mathrm{~mm}^{2}$. The temperature of the holder is measured by an attached K-type thermocouple and is kept close to $290 \mathrm{~K}$. In order to obtain a laterally homogeneous damaged area, the incident beam ( $2 \mathrm{~mm}$ FWHM) is scanned over the whole sample surface. The incident ion flux is controlled during irradiation by four small Faraday cups located at the corners of a water-cooled $\mathrm{Cu}$ diaphragm placed in front of the sample holder. The irradiations are carried out at normal ion incidence and the ion flux is around $10^{16} \mathrm{H} / \mathrm{m}^{2} \mathrm{~s}$. The samples were irradiated to a fluence of $10^{20} \mathrm{H} / \mathrm{m}^{2}$. The depth distribution of the radiation damage (expressed in displacements per atom - dpa) is calculated in SRIM 2013.00 [19] using the "quick" calculation of damage option based on the modified Kinchin-Pease (NRT) model, as recommended by Stoller et al. [20]. The displacement threshold energy of $90 \mathrm{eV}$ [21] is used and the lattice binding energy is set to $0 \mathrm{eV}$. Displacements created both by the incident ions and recoils (listed in the vacancy.txt output file) are taken into account. The calculations predict that most of the displacements $(78 \%)$ are produced directly by the incident ions, which means that only small collision cascades producing mainly Frenkel pairs as primary defects are initiated [22, 23]. The resulting damage profile is non-uniform with a pronounced maximum (Bragg peak) of $5.8 \times 10^{-3}$ dpa located at a depth of $700 \mathrm{~nm}$, as illustrated in Fig. 1. This damage level should be low enough to minimise the formation of complex defect structures due to the overlap of the produced defects [22]. During the irradiation $\mathrm{H}$ ions also get implanted into the material, as shown in Fig. 1. In a fusion reactor $\mathrm{H}$ isotopes will also be present in $\mathrm{W}$ components due to their implantation from plasma and as a product of $(n, p)$ transmutation reactions.

\subsection{Post-irradiation annealing}

After the irradiation, the samples were annealed for $15 \mathrm{~min}$ in vacuum at temperatures in the range of 500-1800 K. A new sample was used for every annealing temperature. Annealing at 500-1300 K was done in a setup where a sample is located in a quartz tube evacuated to about $10^{-6} \mathrm{~Pa}$ 


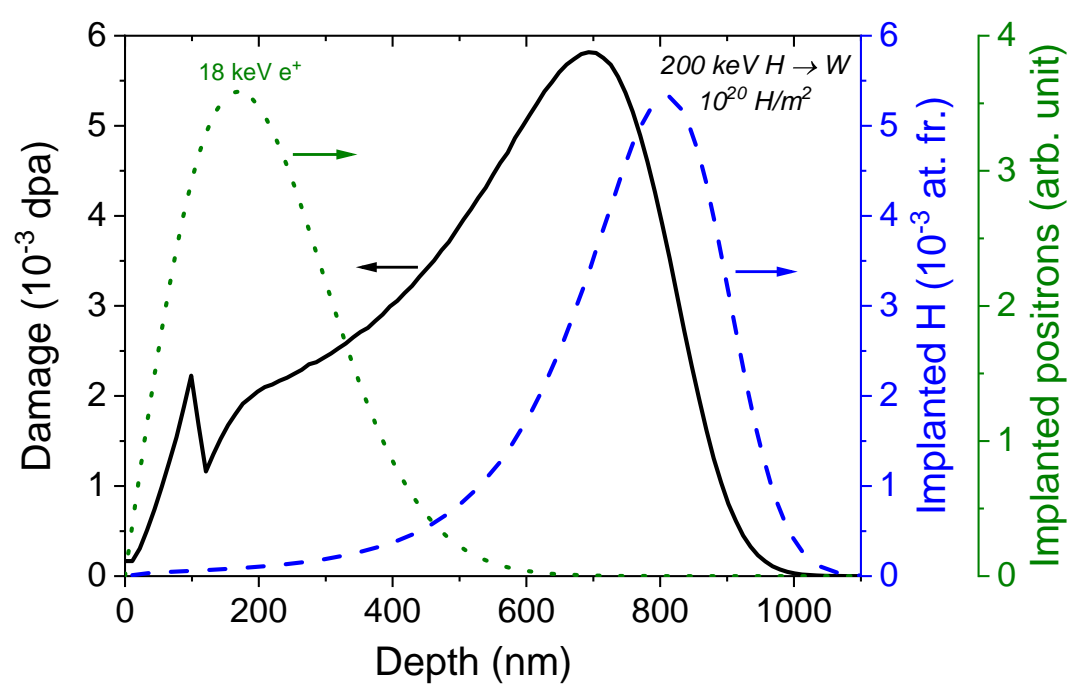

Figure 1: The damage profile created by $200 \mathrm{keV} \mathrm{H}$ ions in $\mathrm{W}$ (solid line) and the $\mathrm{H}$ implantation profile (dashed line) calculated using SRIM. The fluence is $10^{20} \mathrm{H} / \mathrm{m}^{2}$. Note that the peak at a depth of $93 \mathrm{~nm}$ in the damage profile is an artefact due to the free flight path concept of SRIM. The implantation profile of $18 \mathrm{keV}$ positrons in $\mathrm{W}$ (dotted line) calculated according to the Makhov distribution is also shown.

and is heated by radiation from an external tube furnace. The oven is preheated to a certain temperature and then quickly pushed over the tube. At the end of the heating cycle, the furnace is rapidly retracted from the tube. The sample temperature versus the oven temperature is calibrated in independent experiments by a K-type thermocouple spot-welded to the sample. The time required to reach the desired temperature decreases with increasing temperature: it takes around $40 \mathrm{~min}$ to heat the sample from room temperature to $500 \mathrm{~K}$, while only about $2.5 \mathrm{~min}$ are necessary to achieve $1300 \mathrm{~K}$. Annealing at 1400-1800 K was done in a different setup where the samples are located in U-shaped cradles made of $\mathrm{W}$ and heated by $3 \mathrm{keV}$ electron bombardment from the side opposite to the irradiated side at a base pressure below $10^{-6} \mathrm{~Pa}$. The sample temperature is measured with a disappearing-filament pyrometer. The real sample temperature is calculated from the measured brightness temperature using the temperature-dependent W spectral emissivity taken from [24]. The sample temperature was ramped to the desired one within a few minutes. The variations of ramp rates between different samples is not considered important. 


\subsection{Positron annihilation spectroscopy}

Characterization of open-volume defects in the samples, such as vacancies and vacancy clusters, was carried out using two positron annihilation techniques, Doppler broadening spectroscopy (DBS) and positron annihilation lifetime spectroscopy (PALS) [8, 25]. The same samples were measured by both techniques. These methods are based on the fact that positrons implanted into a material exhibit a certain lifetime (before the annihilation with an electron) that depends on the electron density of the medium. Implanted positrons can get trapped at open-volume defects in the lattice. The reduced electron density at such defects increases the lifetime of the positrons. Density functional theory (DFT) calculations indicate that positron lifetimes in vacancy clusters in $\mathrm{W}$ increase with cluster size for clusters containing up to 20 vacancies and then tend to saturate for larger clusters [26]. In addition, the momentum of the annihilating electron creates a Doppler shift in the energy of the two $511 \mathrm{keV}$ annihilation $\gamma$-quanta. The lack of high-momentum core electrons at open-volume defects reduces the average Doppler shift as compared with the defect-free material, i.e., narrows the annihilation line. Hence, $\gamma$-quanta carry information about the environment of the annihilating positron.

The DBS measurements were carried out using a monoenergetic positron beam produced by a moderated ${ }^{22} \mathrm{Na}$ source (Aalto University). To perform depth profiling of the defects, the incident positron energy is varied in the range of $0.5-25 \mathrm{keV}$ corresponding to mean implantation depths in $\mathrm{W}$ in the range of 0.7-360 $\mathrm{nm}$ and a maximum information depth of $700 \mathrm{~nm}$ [25]. A high-purity germanium detector with good energy resolution (FWHM = $1.2 \mathrm{keV}$ at $511 \mathrm{keV}$ ) and Gaussian response is used for the Doppler broadening recordings. The number of collected counts is $10^{6}$. The broadening is characterized with the annihilation parameters $S$ and $W$ that represent the fraction of annihilations with low-momentum and high-momentum electrons, respectively. The $S$ parameter is defined as the number of counts in the central region of the annihilation line (0-0.41 a.u.; 0-0.76 keV) divided by the total number of counts in the annihilation line. $W$ is defined as the counts in the wing regions of the annihilation line (1.6-4.0 a.u.; $2.9-7.5 \mathrm{keV}$ ) divided by the total number of counts in the annihilation line. The presence of openvolume defects in the material results in an increase of $S$ and a respective decrease of the $W$ parameter. Furthermore, the $S-W$ correlation plots can be used to identify the defect types [25]. The positron diffusion lengths in the samples are determined by analysing the measured $S(E)$ dependences 
using VEPFIT [27.

The PALS measurements were carried out using the pulsed low-energy positron beam system (PLEPS) [28, 29] at the NEPOMUC positron source (FRM II reactor). The system utilizes a pulsed positron beam (150 ps FWHM, frequency $50 \mathrm{MHz}$ ) with high intensity and a low background. The used positron implantation energy is $18 \mathrm{keV}$. The positron implantation profile at this energy is shown in Fig. 1. The $\gamma$-quanta are detected with a photomultiplier coupled to a $\mathrm{BaF}_{2}$ scintillator and the time between the annihilation and the following pulse is recorded. The total number of events collected in each lifetime spectrum is $4 \times 10^{6}$. The instrumental time resolution function $R(t)$, which can be described by a sum of three-four shifted Gaussian functions, is determined with a p-type $\mathrm{SiC}$ reference sample and has a FWHM of 230-250 ps. The measured data are analysed using POSWIN (a modified version of PositronFit [30]) where the model function is fitted to an experimental spectrum. The model function is described by a sum of exponential decays convoluted with $R(t)$ and overlaid on a constant background $B G: R(t) * \sum_{i}\left(I_{i} / \tau_{i}\right) \exp \left(-t / \tau_{i}\right)+B G$, where $\tau_{i}$ and $I_{i}\left(\sum_{i} I_{i}=1\right)$ represent positron lifetimes and their relative intensities, respectively. Apart from the $\tau_{1}$ component due to positrons annihilating in the defect-free bulk, each defect type gives rise to a characteristic lifetime $\tau_{i}(i=2,3, \ldots)$ in a lifetime spectrum. $I_{i}$ is a measure of the relative concentration of the defects associated with $\tau_{i}$. Practically, components with lifetimes close to each other cannot be resolved. Therefore, the lifetime components extracted from experimental spectra can sometimes represent averaged "effective" values. In the present work the spectra were decomposed into three to four lifetime components with good variance $\left(\chi_{\nu}^{2}<1.6\right)$. The intensity of the fourth lifetime is always below $1 \%$, therefore, it will be neglected in the discussion and the third lifetime will be always referred to as the longest lifetime. Although the decomposition of the spectra into lifetime components may be sometimes ambiguous due to the extreme sensitivity of exponential fits to noise in the data [31], the average positron lifetime $\tau_{a v}=\sum_{i} I_{i} \tau_{i}$ representing the statistical mean of the lifetime distribution is almost insensitive to uncertainties of the decomposition. 


\section{Results and discussion}

\subsection{Qualitative analysis}

The recorded positron lifetime spectra shown in Fig. 2 clearly demonstrate significant differences in the positron lifetimes, and, hence, in the defect structure of the samples after irradiation and subsequent annealing at various temperatures. To assess the reproducibility of the sample preparation procedures, two unirradiated and two as-irradiated specimens have been measured and in both cases the lifetime spectra of two samples are almost indistinguishable. To reduce the statistical scatter in the data, the averaged PALS results over two unirradiated and as-irradiated samples will be shown.

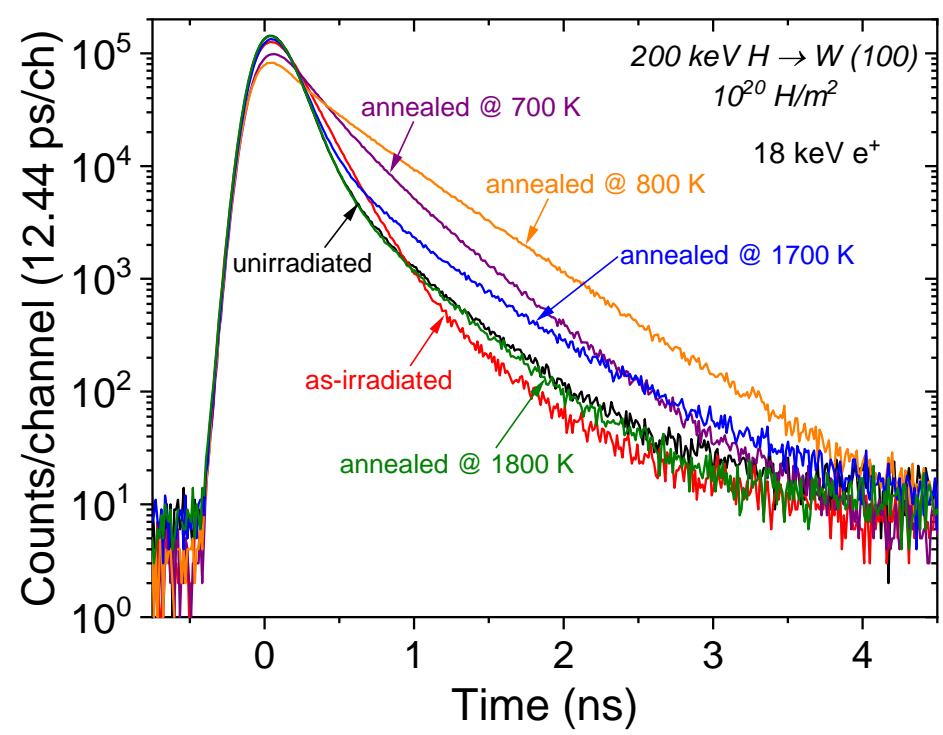

Figure 2: Positron lifetime spectra at $18 \mathrm{keV}$ positron implantation energy for W (100) single crystals irradiated with $200 \mathrm{keV} \mathrm{H}$ ions to a fluence of $10^{20} \mathrm{H} / \mathrm{m}^{2}$ and then annealed at various temperatures for $15 \mathrm{~min}$.

A general idea about the evolution of the defect structure with annealing temperature can be obtained from the changes of the average positron lifetime $\tau_{a v}$ and of the $S$ parameter. These are shown in Fig. 3 for $18 \mathrm{keV}$ positrons. Both $\tau_{a v}$ and $S$ demonstrate remarkably similar behaviour, which shows that they describe similar characteristics of the defects. An unirradiated sample has the lowest $\tau_{a v}$ close to the typical defect-free bulk lifetime, indicating that the majority of the positrons annihilate from the delocalized 
bulk state. Irradiation with $200 \mathrm{keV} \mathrm{H}$ ions increases the $S$ parameter and $\tau_{a v}$

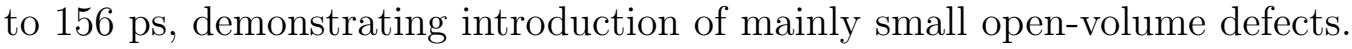
Post-irradiation annealing at $700 \mathrm{~K}$ and $800 \mathrm{~K}$ results in a sharp increase of $\tau_{a v}$ and $S$, indicating trapping of positrons in large open-volume defects, such as vacancy clusters. Both parameters then weakly decrease after annealing at temperatures in the range of 900-1200 K. Starting from $1300 \mathrm{~K}$ both $\tau_{a v}$ and $S$ decrease considerably with increasing temperature. This can be attributed to progressive defect recovery. Eventually, after annealing at $1800 \mathrm{~K}$ both parameters are identical to those of the unirradiated sample, demonstrating complete removal of radiation defects.

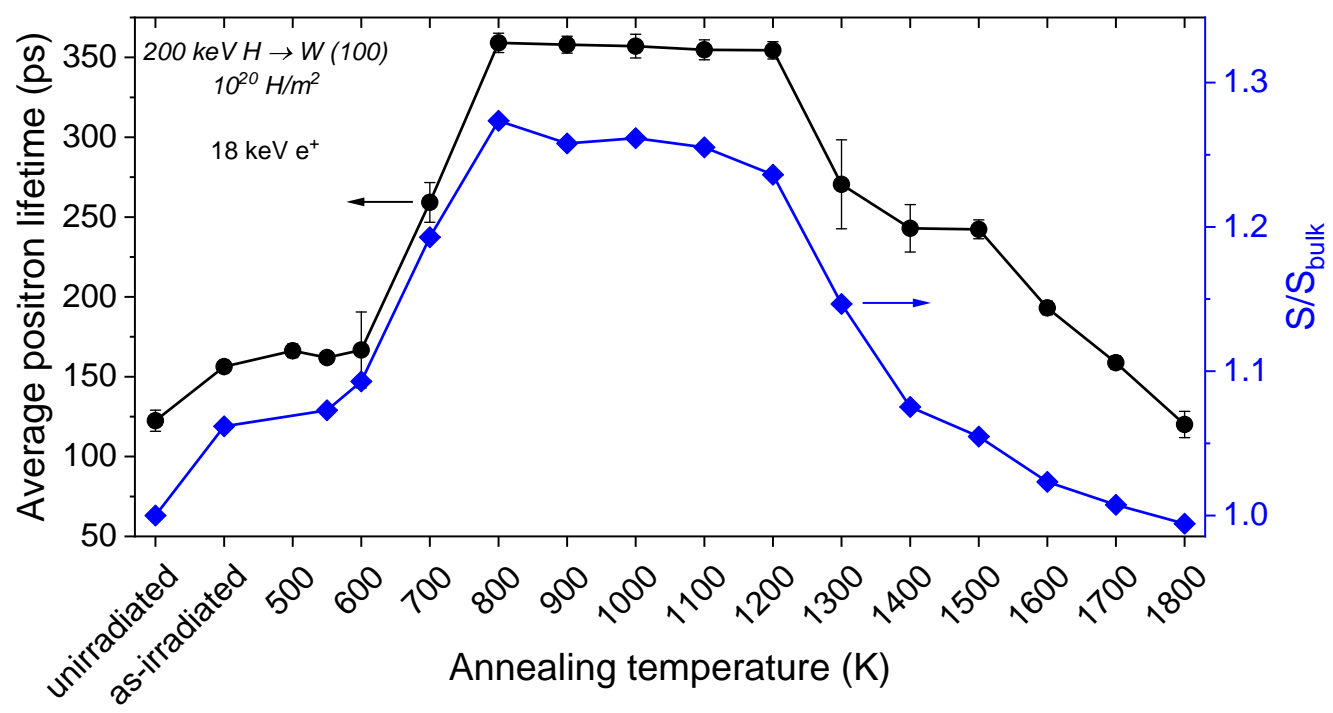

Figure 3: Average positron lifetime $\left(\tau_{a v}\right)$ and low-momentum annihilation fraction $(S)$ as function of annealing temperature for W (100) single crystals irradiated with $200 \mathrm{keV} \mathrm{H}$ ions to a fluence of $10^{20} \mathrm{H} / \mathrm{m}^{2}$ and then annealed at various temperatures for $15 \mathrm{~min}$. The positron implantation energy is $18 \mathrm{keV}$. For the unirradiated sample and the samples annealed at $1600 \mathrm{~K}, 1700 \mathrm{~K}$, and $1800 \mathrm{~K}$ the corresponding bulk $S$ parameter values are obtained from VEPFIT because of large positron diffusion lengths in the samples. The $S$ parameter is normalized to the bulk value of the unirradiated sample $\left(S_{\text {bulk }}\right)$ obtained from VEPFIT.

A similar temperature dependence of the $S$ parameter is observed at positron implantation energies starting from about $12 \mathrm{keV}$ (Fig. 4). At lower energies the $S$ parameter is influenced by surface effects: the lower the implantation energy is, the higher is the probability for a positron to diffuse back to the surface where it can either annihilate or escape from the material. 


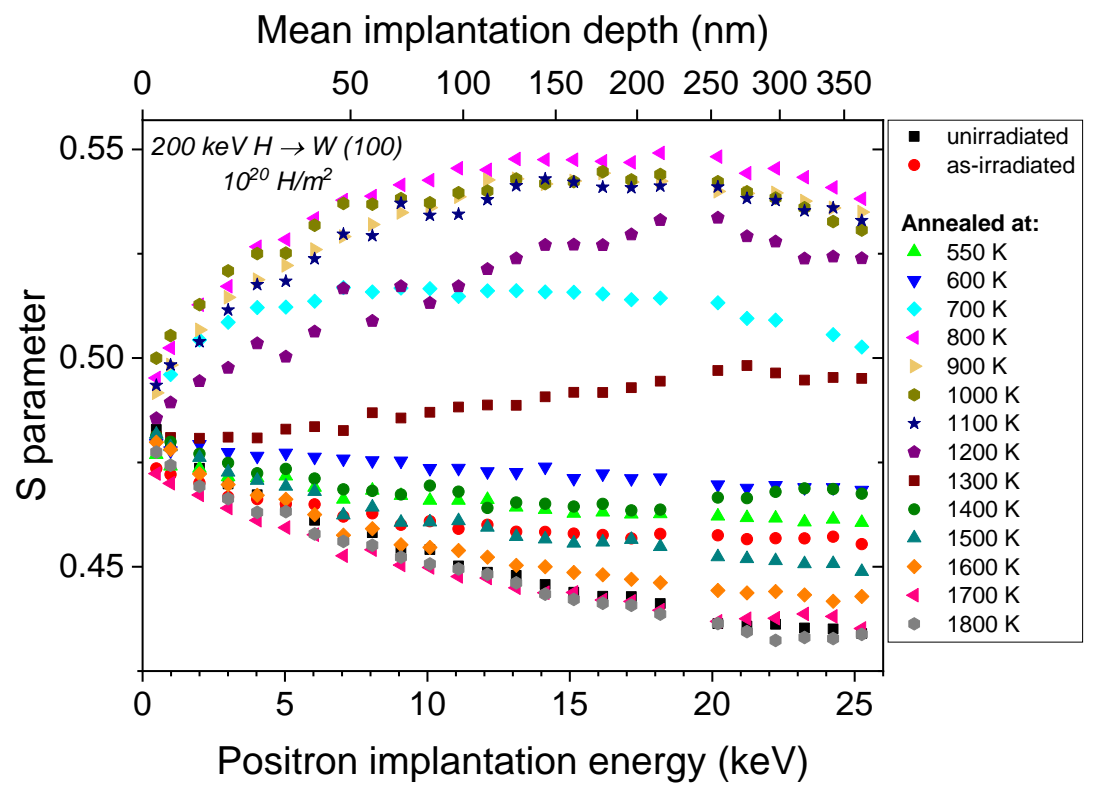

Figure 4: Low-momentum annihilation fraction $(S)$ as function of the positron implantation energy in $\mathrm{W}(100)$ single crystals irradiated with $200 \mathrm{keV} \mathrm{H}$ ions to a fluence of $10^{20} \mathrm{H} / \mathrm{m}^{2}$ and then annealed at various temperatures for $15 \mathrm{~min}$. The top $x$-axis shows the corresponding mean positron implantation depth calculated according to the Makhov distribution.

\subsection{Quantitative analysis}

More detailed insight into the changes of the defect sizes and concentrations can be gained from the results of the decomposition of the positron lifetime spectra into components shown in Fig. 5. Thanks to the peak-tobackground ratio in the spectra above $10^{4}$ (Fig. 2) and no need in subtracting annihilations in the source, long lifetimes with even small intensities can be observed.

\subsubsection{Reference material}

Measuring the unirradiated samples using a conventional fast positron lifetime spectrometer yields a single lifetime of $110 \mathrm{ps}$, corresponding to the bulk lifetime in W (100-110 ps) [13, 26, 32, 33, 34, 35]. Spectra of unirradiated samples measured with $18 \mathrm{keV}$ pulsed positron beam can be decomposed with a good variance into three lifetime components. The shortest lifetime ("reduced bulk lifetime") comprises both the annihilation from the delocalized bulk state and disappearance from it by trapping at the surface (or 
escaping from the material). DBS data shows that the positron diffusion length (before the annihilation) in the unirradiated sample is $96 \mathrm{~nm}$, which is characteristic for a well-annealed W [11]. As the $S(E)$ profile of the unirradiated specimen has not reached the plateau even at $25 \mathrm{keV}$ (Fig. 4), the fraction of positrons implanted with $18 \mathrm{keV}$ that diffuse back to the surface is not negligible. Therefore, the longest positron lifetime near 350 ps should correspond to annihilation at the $\mathrm{W}$ surface. The reported positron lifetimes at metallic surfaces are lying in a very wide range of 350-600 ps [32, 36, 37]. The second lifetime is around 100 ps, corresponding to the defect-free (bulk) lifetime in $\mathrm{W}[13,26,32,33,34,35]$.

\subsubsection{Initial defect configuration}

In the as-irradiated samples almost $80 \%$ of positrons are trapped in defects with a lifetime of 165 ps. This value is slightly lower than the reported positron lifetime in a single vacancy in $W(180-200 \mathrm{ps})$ [11, 13, 14, 15, 26, 33, 34, 35, 38. This can be due to the fact that the vacancies in the asirradiated samples are (at least partly) filled with implanted $\mathrm{H}$, which decreases the positron lifetime in them [14, 26, 38]. Neglecting the presence of other defect types, the fraction of positrons annihilating in these dominant defects can be estimated using the determined positron diffusion lengths in unirradiated $\left(L_{r e f}=96 \mathrm{~nm}\right)$ and as-irradiated samples $\left(L_{i r r}=45 \mathrm{~nm}\right)$ as $\eta_{V}=\left(L_{r e f}^{2}-L_{i r r}^{2}\right) / L_{r e f}^{2}=0.78$ [39]. It agrees well with the relative intensity of these defects in the lifetime spectra. Using the bulk relative $S$ and $W$ values for the as-irradiated sample, the bulk relative $S_{V}$ and $W_{V}$ values characteristic for the dominant defect type in the as-irradiated sample (presumably single vacancies) were obtained. Less than $5 \%$ of positrons in the as-irradiated samples annihilate in defects with a lifetime around $420 \mathrm{ps}$. Due to a smaller positron diffusion length in this sample $(45 \mathrm{~nm})$ and the fact that the respective $S(E)$ profile has already reached a plateau at $18 \mathrm{keV}$, the fraction of implanted positrons annihilating at the surface should be rather small. Consequently, the 420 ps component should mainly correspond to annihilation at vacancy clusters introduced during irradiation.

\subsubsection{Defect behaviour during annealing}

500-600 K. Post-irradiation annealing at temperatures in the range of 500$600 \mathrm{~K}$ results in a gradual increase of the second lifetime up to 195 ps. This value is within the range of reported positron lifetimes in an empty single vacancy in $\mathrm{W}$. The value of the longest lifetime fluctuates around $420 \mathrm{ps}$, 


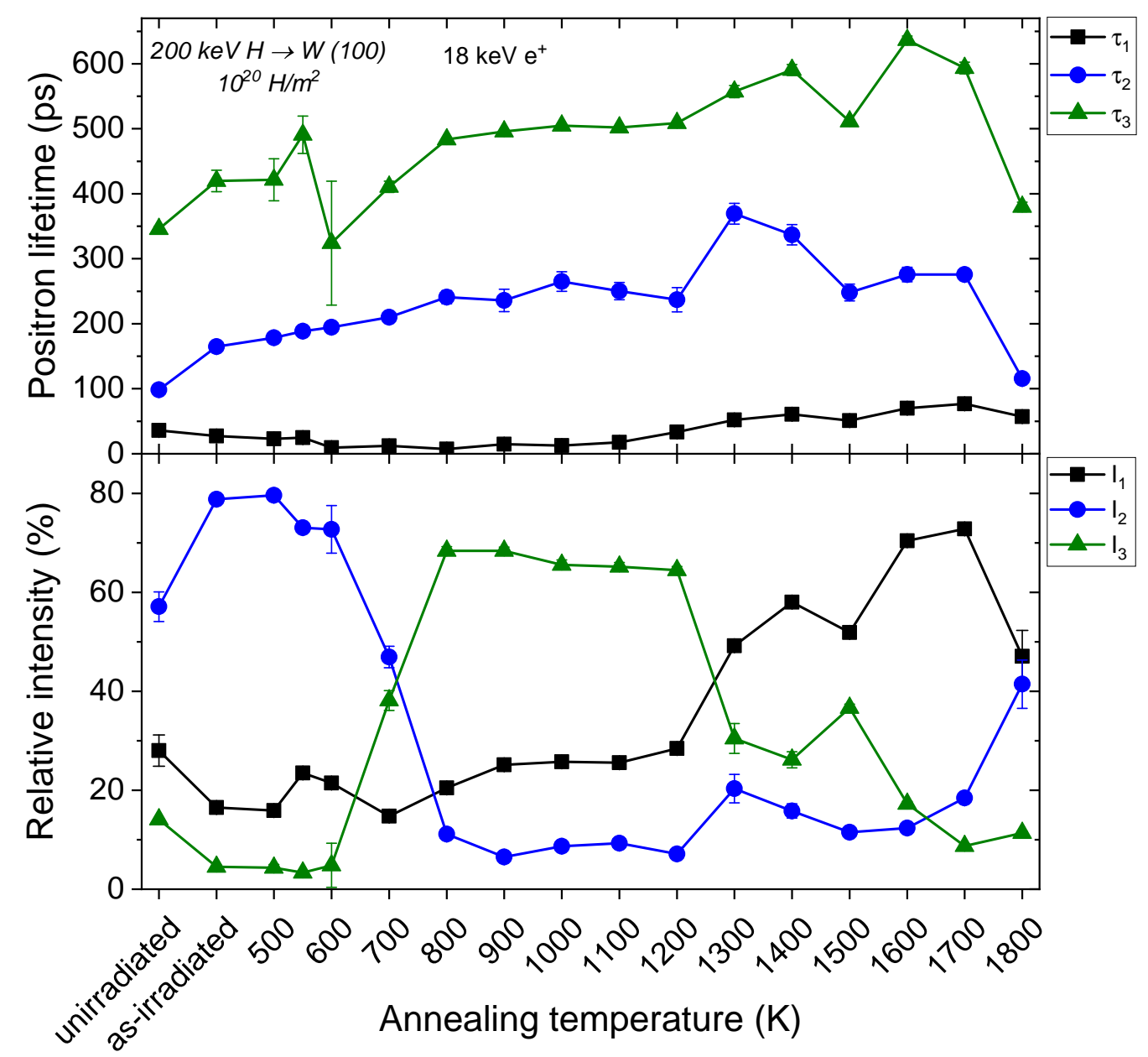

Figure 5: Positron lifetimes (upper panel) and their relative intensities (lower panel) as a function of annealing temperature for W (100) single crystals irradiated with $200 \mathrm{keV}$ $\mathrm{H}$ ions to a fluence of $10^{20} \mathrm{H} / \mathrm{m}^{2}$ and then annealed at various temperatures for $15 \mathrm{~min}$. The positron implantation energy is $18 \mathrm{keV}$.

while its intensity stays within $5 \%$ indicating little changes in the structure of the existing vacancy clusters. In the $S-W$ map shown in Fig. 6 the points corresponding to the as-irradiated sample and the samples annealed at 500$600 \mathrm{~K}$ are lying on the same line (1) connecting the bulk $S$ - $W$ value with the $S_{V}-W_{V}$ value characteristic for the introduced defects (presumably single vacancies). This demonstrates that the nature of the defects does not change 
significantly in this temperature range. The observed increase of the second lifetime is likely due to the release of trapped $\mathrm{H}$ from vacancies, which is known to occur in this temperature range [9, 14, 40].

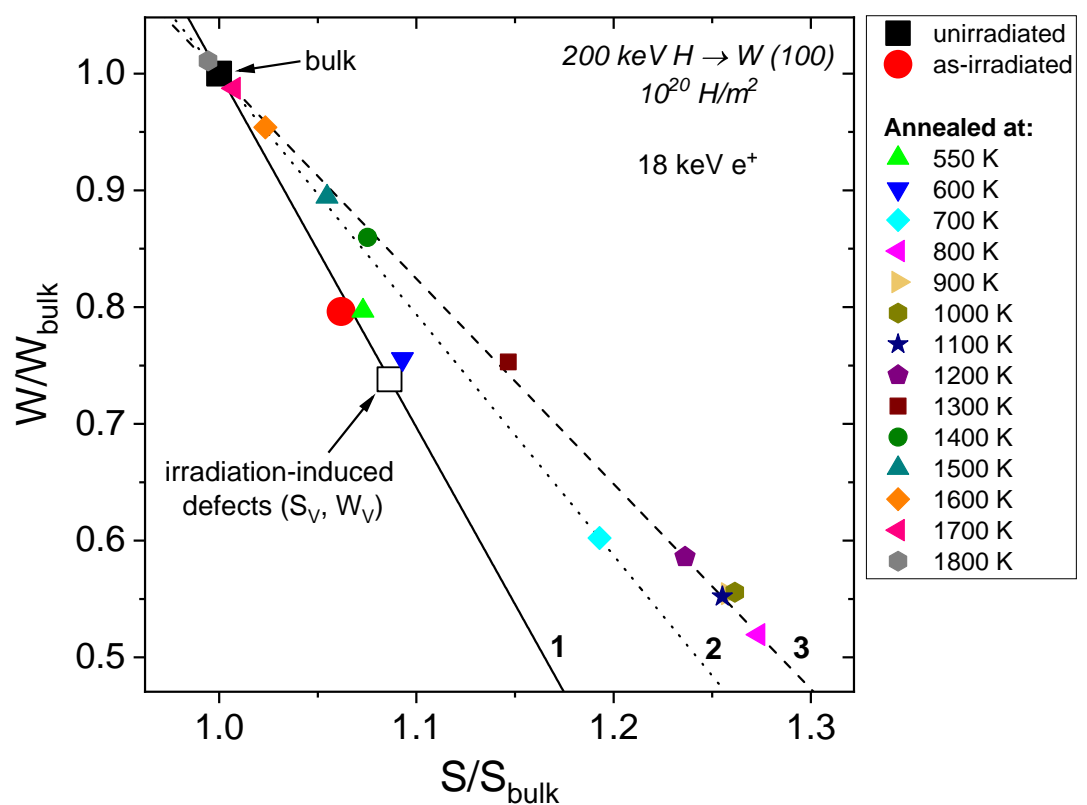

Figure 6: The $S$ - $W$ plot for W (100) single crystals irradiated with $200 \mathrm{keV} \mathrm{H}$ ions to a fluence of $10^{20} \mathrm{H} / \mathrm{m}^{2}$ and then annealed at various temperatures for $15 \mathrm{~min}$. The positron implantation energy is $18 \mathrm{keV}$. For the unirradiated sample and the samples annealed at $1600 \mathrm{~K}, 1700 \mathrm{~K}$, and $1800 \mathrm{~K}$ the corresponding bulk $S$ parameter values are obtained from VEPFIT because of large positron diffusion lengths in the samples. The shown parameters are normalized to the bulk values for the unirradiated sample $\left(S_{\text {bulk }}, W_{\text {bulk }}\right)$ obtained from VEPFIT. The open square shows the calculated $\left(S_{V}, W_{V}\right)$ for the dominant defect type in the as-irradiated sample (presumably single vacancy) based on the determined positron annihilation fraction $\eta_{V}$ in them. The lines indicate positron annihilation at different defect types: (1) single vacancies, (2) medium-size vacancy clusters, (3) large vacancy clusters.

$700-1200 \mathrm{~K}$. Annealing at $700 \mathrm{~K}$ and $800 \mathrm{~K}$ results in a sharp increase of the intensity (and some increase of the value) of the longest lifetime component. This is accompanied by a decrease of the intensity of the second lifetime. After annealing at $700 \mathrm{~K}$ about half of the positrons annihilate in defects with a lifetime of 210 ps representing the mixture of annihilations at vacancies and small vacancy clusters; almost $40 \%$ of positrons annihilate at defects with a 
lifetime of 410 ps corresponding to clusters containing 10-15 vacancies [26]. In the temperature range of $800-1200 \mathrm{~K}$ the lifetime components change only slightly. The dominant (around 65\%) longest lifetime close to 500 ps demonstrates the presence of clusters containing about 20 vacancies or more [26] and represents the theoretical limit of the positron lifetime in a cavity in a metal [33. In the $S$ - $W$ plot (Fig. 6) it is clearly visible that the data from the sample annealed at $700 \mathrm{~K}$ does not lie on the line (1). After annealing at $800 \mathrm{~K}$ and higher temperatures, all data points lie close to the line (3). The smaller slope of line (3) as compared with (1) indicates the larger open volume associated with the dominant defects, in agreement with PALS results.

Despite the rather convincing results described above, their interpretation requires some care. It should be kept in mind that neither PALS nor DBS techniques are able to distinguish between the growth of vacancy clusters and $\mathrm{H}$ release from the vacancy clusters created during the irradiation as both processes increase the effective open volume [14, 26, 36]. The observed increase of the relative intensity of vacancy clusters accompanied by the decrease of the intensity of vacancies in the PALS spectra after annealing at $700 \mathrm{~K}$ and $800 \mathrm{~K}$ can be explained by the formation of vacancy clusters at the expense of single vacancies. This process is driven by the onset of mobility of vacancies and corresponds to recovery stage III. The reduced bulk lifetime $\tau_{1}$ first decreases after annealing at 550-800 K and then increases at higher temperatures. This indicates that after annealing up to $800 \mathrm{~K}$ the total positron trapping rate into the defects, which reflects the total concentration of the defects, increases. Note that for vacancy clusters the trapping rate per missing atom typically decreases with increasing vacancy cluster size [36]. Therefore, the apparent total open volume where the positrons annihilate likely increases after annealing at 500-800 K. This is correlated with the $\mathrm{H}$ release from vacancies and vacancy clusters occurring in this temperature range [9, 40, 41, 42]. Above $800 \mathrm{~K}$ all trapped $\mathrm{H}$ is expected to be removed from the defects and there is no further influence of the presence of $\mathrm{H}$ on the thermal evolution of the defects.

The present results show that annealing already at $800 \mathrm{~K}$ results in the predominant presence of clusters containing about 20 or more vacancies. A similar result has also been reported by de Vries [14]. The cluster sizes observed after annealing at $700 \mathrm{~K}$ and $800 \mathrm{~K}$ are larger than the smallest cluster sizes that are still thermally stable at the corresponding temperatures according to theoretical estimations [9, 43] and Kinetic Monte Carlo simulations [44]. The theoretical estimations are based on the assumption 
that the cluster thermal dissociation occurs via emission of single vacancies from it (first-order Arrhenius-like process) and are using calculated vacancy binding energies to a cluster as a function of cluster size [43]. This observation can be explained by the fact that cluster coarsening with increasing annealing temperature is influenced not only by the migration and agglomeration of single vacancies ("Ostwald ripening"), but also by the mobility and coalescence of small vacancy clusters [7, 45].

1300-1800 K. After annealing at $1300 \mathrm{~K}$ and higher temperatures, the fraction of positrons annihilating in the bulk starts to increase considerably, indicating defect recovery. The longest lifetime increases up to about 600 ps while its intensity decreases. This lifetime is above the theoretical limit of 500 ps described above and may correspond to pick-off annihilation of orthopositronium formed in large vacancy clusters. This suggests that the internal surfaces of the clusters may be partially decorated with impurities such as $\mathrm{O}$ and $\mathrm{C}$ [36]. Hu et al. [46] reported the formation of voids with a diameter around $1 \mathrm{~nm}$ visible in TEM after post-irradiation annealing at $1273 \mathrm{~K}$ of a W single crystal irradiated by fission neutrons to $3 \times 10^{-2}$ dpa. Therefore, it is possible that in the present experiments further coarsening of vacancy clusters accompanied by the segregation of impurities at their surfaces took place at $1300 \mathrm{~K}$ and higher temperatures. This corresponds to the recovery stage $\mathrm{V}$. The intermediate stage (IV) is not pronounced in the present experiments, which means that little changes of open-volume defects occur during it. This stage can correspond to annealing of interstitial-type dislocation loops, as was proposed by $\mathrm{Hu}$ et al. [46].

After annealing at $1800 \mathrm{~K}$ the lifetime components are the same as in the unirradiated sample, albeit the intensities of the first and second components are somewhat different. This temperature is above the expected operation temperature of $\mathrm{W}$ components in the ITER divertor and is also higher than the recrystallization temperature of $\mathrm{W}[2,3]$.

\section{Summary}

An investigation of the thermal evolution of vacancies and vacancy clusters in single crystalline $\mathrm{W}$ in the temperature range 500-1800 K has been carried out. The introduction of predominantly Frenkel pairs as primary defects was done by irradiation with $200 \mathrm{keV} \mathrm{H}$ ions to a low damage level $\left(5.8 \times 10^{-3} \mathrm{dpa}\right)$. The evolution of the sizes and concentrations of the defects was investigated using PALS and DBS techniques. 
Substantial changes in the defect structure were observed after postirradiation annealing at $700 \mathrm{~K}$ and were driven by the onset of vacancy mobility, resulting in their agglomeration into clusters containing 10-15 vacancies. Annealing at $800 \mathrm{~K}$ and above resulted in the formation of large clusters containing about 20 or more vacancies, a size which cannot be further distinguished by PALS. In the temperature range of 800-1200 K the concentration of open-volume defects practically did not change, whereas at temperatures of $1300 \mathrm{~K}$ and above recovery of the defects started and is likely accompanied by a further coarsening of the vacancy clusters. The complete removal of radiation defects was observed after annealing at $1800 \mathrm{~K}$. The determined sizes of the clusters after annealing at $700 \mathrm{~K}$ and $800 \mathrm{~K}$ were larger than the minimal sizes expected from thermal stability considerations, therefore, it is suggested that the mobility of small vacancy clusters plays an important role in the cluster growth.

\section{Acknowledgements}

The technical assistance of T. Dürbeck, K. Eismann, J. Dorner, and M. Fußeder is gratefully acknowledged. We would also like to thank H. Brongersma and V. Glebovsky for providing the W single crystals, A. Manhard and K. Schlüter for the help with the sample preparation, and B. Wielunska for the Laue diffraction measurements of the samples. J.H. and F.T. acknowledge the financial support from the Academy of Finland project Nr. 315082. This work was supported by the European Commission and carried out within the framework of the Erasmus Mundus International Doctoral College in Fusion Science and Engineering (FUSION-DC). This work has also been carried out within the framework of the EUROfusion Consortium and has received funding from the Euratom research and training programme 2014-2018 and 2019-2020 under grant agreement No 633053. The views and opinions expressed herein do not necessarily reflect those of the European Commission.

\section{References}

[1] J. Knaster, A. Moeslang, T. Muroga, Materials research for fusion, Nature Physics 12 (2016) 424.

URL http://dx.doi .org/10.1038/nphys3735 
[2] Y. Ueda, K. Schmid, M. Balden, J. Coenen, T. Loewenhoff, A. Ito, A. Hasegawa, C. Hardie, M. Porton, M. Gilbert, Baseline high heat flux and plasma facing materials for fusion, Nuclear Fusion 57 (9) (2017) 092006.

URL http: //stacks . iop.org/0029-5515/57/i=9/a=092006

[3] M. Rieth, R. Doerner, A. Hasegawa, Y. Ueda, M. Wirtz, Behavior of tungsten under irradiation and plasma interaction, Journal of Nuclear Materials 519 (2019) 334 - 368. doi:10.1016/j.jnucmat.2019.03.035. URL http://www.sciencedirect.com/science/article/pii/ S002231151930025X

[4] H. Ullmaier (Ed.), Landolt-Börnstein - Group III Condensed Matter. Volume 25: "Atomic Defects in Metals", Springer-Verlag Berlin Heidelberg, 1991. doi:10.1007/b37800.

[5] S. Zinkle, Radiation-Induced Effects on Microstructure, Elsevier, Oxford, 2012, pp. 65-98. doi:10.1016/B978-0-08-056033-5.00003-3.

URL http://www.sciencedirect.com/science/article/pii/ B9780080560335000033

[6] F. Ferroni, X. Yi, K. Arakawa, S. P. Fitzgerald, P. D. Edmondson, S. G. Roberts, High temperature annealing of ion irradiated tungsten, Acta Materialia 90 (2015) 380 - 393. doi:10.1016/j . actamat.2015.01.067. URL http://wWw.sciencedirect.com/science/article/pii/ S1359645415000804

[7] A. De Backer, P. Lhuillier, C. Becquart, M. Barthe, Modelling of the implantation and the annealing stages of $800 \mathrm{keV}{ }^{3} \mathrm{He}$ implanted tungsten: Formation of nanovoids in the near surface region, Journal of Nuclear Materials 429 (1) (2012) 78 - 91. doi:10.1016/j.jnucmat.2012.05. 024 .

[8] M. Eldrup, B. Singh, Studies of defects and defect agglomerates by positron annihilation spectroscopy, Journal of Nuclear Materials 251 (1997) 132 - 138. doi:10.1016/S0022-3115(97)00221-3.

URL http://www.sciencedirect.com/science/article/pii/ S0022311597002213 
[9] H. Eleveld, A. van Veen, Void growth and thermal desorption of deuterium from voids in tungsten, Journal of Nuclear Materials 212-215 (1994) 1421 - 1425. doi:10.1016/0022-3115(94)91062-6.

URL http://www.sciencedirect.com/science/article/pii/ 0022311594910626

[10] A. Debelle, M. Barthe, T. Sauvage, First temperature stage evolution of irradiation-induced defects in tungsten studied by positron annihilation spectroscopy, Journal of Nuclear Materials 376 (2) (2008) 216 - 221. doi:10.1016/j.jnucmat.2008.03.002.

URL http://www.sciencedirect.com/science/article/pii/ S0022311508001724

[11] P. E. Lhuillier, M. F. Barthe, P. Desgardin, W. Egger, P. Sperr, Positron annihilation studies on the nature and thermal behaviour of irradiation induced defects in tungsten, Physica Status Solidi C 6 (11) (2009) 23292332. doi:10.1002/pssc.200982114.

URL http://dx.doi.org/10.1002/pssc. 200982114

[12] K. Sato, R. Tamiya, Q. Xu, H. Tsuchida, T. Yoshiie, Detection of deuterium trapping sites in tungsten by thermal desorption spectroscopy and positron annihilation spectroscopy, Nuclear Materials and Energy 9 (2016) 554 - 559. doi:10.1016/j.nme.2016.09.014.

URL http://www.sciencedirect.com/science/article/pii/ S2352179115300764

[13] J. Heikinheimo, K. Mizohata, J. Räisänen, T. Ahlgren, P. Jalkanen, A. Lahtinen, N. Catarino, E. Alves, F. Tuomisto, Direct observation of mono-vacancy and self-interstitial recovery in tungsten, APL Materials 7 (2) (2019) 021103. doi:10.1063/1.5082150.

[14] J. de Vries, Positron lifetime technique with applications in materials science, Ph.D. thesis, Technische Universiteit Delft, The Netherlands (1987).

URL http://resolver.tudelft.nl/uuid: c2641211-ecfe-491e-9ee9-5f2cbb5ba8b8

[15] O. Ogorodnikova, L. Y. Dubov, S. Stepanov, D. Terentyev, Y. Funtikov, Y. Shtotsky, V. Stolbunov, V. Efimov, K. Gutorov, Annealing of 
radiation-induced defects in tungsten: Positron annihilation spectroscopy study, Journal of Nuclear Materials 517 (2019) 148 - 151. doi:10.1016/j.jnucmat.2019.02.010.

URL http://wWW.sciencedirect.com/science/article/pii/ S0022311518313655

[16] V. Glebovsky, V. Semenov, V. Lomeyko, Influence of the crystallization conditions on the structural perfection of molybdenum and tungsten single crystals, Journal of Crystal Growth 87 (1) (1988) 142-150. doi:10.1016/0022-0248(88)90353-3.

URL http://www.sciencedirect.com/science/article/pii/ 0022024888903533

[17] V. Glebovsky, Crystal Growth: Substructure and Recrystallization, InTech, 2012, p. 59. doi:10.5772/34871.

[18] T. Schwarz-Selinger, Deuterium retention in MeV self-implanted tungsten: Influence of damaging dose rate, Nuclear Materials and Energy 12 (2017) 683 - 688. doi:10.1016/j.nme.2017.02.003.

URL http://www.sciencedirect.com/science/article/pii/ S2352179116301922

[19] J. F. Ziegler, J. P. Biersack, M. D. Ziegler, SRIM - The Stopping and Range of Ions in Matter, www.srim.org, SRIM co., Chester, Maryland, USA, 2008.

URL http: //www . srim.org/

[20] R. Stoller, M. Toloczko, G. Was, A. Certain, S. Dwaraknath, F. Garner, On the use of SRIM for computing radiation damage exposure, Nuclear Instruments and Methods in Physics Research Section B 310 (2013) 75 - 80. doi:10.1016/j.nimb.2013.05.008.

URL http://www.sciencedirect.com/science/article/pii/ S0168583X13005053

[21] Standard practice for investigating the effects of neutron radiation damage using charged-particle irradiation, Tech. Rep. ASTM E521-16, ASTM International, West Conshohocken, Pennsylvania, USA (2016). doi : 10.1520/E0521-16. 
[22] C. Abromeit, Aspects of simulation of neutron damage by ion irradiation, Journal of Nuclear Materials 216 (1994) 78 - 96. doi:10.1016/0022-3115(94)90008-6.

URL http://www.sciencedirect.com/science/article/pii/ 0022311594900086

[23] G. Was, R. Averback, 1.07 - Radiation Damage Using Ion Beams, Elsevier, Oxford, 2012, pp. 195 - 221. doi:10.1016/B978-0-08-056033-5. 00007-0.

URL http://www.sciencedirect.com/science/article/pii/ B9780080560335000070

[24] S. Yih, C. Wang, Tungsten: sources, metallurgy, properties, and applications, Plenum Press, New York, 1979.

[25] F. Tuomisto, I. Makkonen, Defect identification in semiconductors with positron annihilation: Experiment and theory, Rev. Mod. Phys. 85 (2013) 1583-1631. doi:10.1103/RevModPhys.85.1583.

[26] T. Troev, E. Popov, P. Staikov, N. Nankov, T. Yoshiie, Positron simulations of defects in tungsten containing hydrogen and helium, Nuclear Instruments and Methods in Physics Research Section B 267 (3) (2009) 535 - 541. doi:10.1016/j.nimb.2008.11.045.

URL http://www.sciencedirect.com/science/article/pii/ S0168583X08013190

[27] A. van Veen, H. Schut, M. Clement, J. de Nijs, A. Kruseman, M. IJpma, Vepfit applied to depth profiling problems, Applied Surface Science 85 (1995) 216 - 224. doi:10.1016/0169-4332(94)00334-3.

[28] P. Sperr, W. Egger, G. Kögel, G. Dollinger, C. Hugenschmidt, R. Repper, C. Piochacz, Status of the pulsed low energy positron beam system (PLEPS) at the Munich research reactor FRM-II, Applied Surface Science 255 (2008) 35 - 38. doi :10.1016/j .apsusc.2008.05.307. URL http://www.sciencedirect.com/science/article/pii/ S016943320801218X

[29] W. Egger, Pulsed low energy positron beams in materials sciences, IOS Press, Amsterdam, 2010, p. 419. doi:10.3254/ 978-1-60750-647-8-419. 
[30] P. Kirkegaard, J. Olsen, M. Eldrup, PALSfit3: A software package for analysing positron lifetime spectra, Tech. rep., Technical University of Denmark (DTU) (2017).

URL http://orbit.dtu.dk/en/publications/ palsfit3-a-software-package-for-analysing-positron-lifetime-spectra(7e984e17.html

[31] A. A. Istratov, O. F. Vyvenko, Exponential analysis in physical phenomena, Review of Scientific Instruments 70 (2) (1999) 1233-1257. doi:10.1063/1.1149581. URL https://doi.org/10.1063/1.1149581

[32] T. E. M. Staab, R. Krause-Rehberg, B. Vetter, B. Kieback, G. Lange, P. Klimanek, The influence of microstructure on the sintering process in crystalline metal powders investigated by positron lifetime spectroscopy: Ii. tungsten powders with different powder-particle sizes, Journal of Physics: Condensed Matter 11 (7) (1999) 1787.

URL http://stacks . iop.org/0953-8984/11/i=7/a=010

[33] A. Seeger, F. Banhart, On the systematics of positron lifetimes in metals, physica status solidi (a) 102 (1) (1987) 171-179. doi:10.1002/pssa.2211020117.

URL https://onlinelibrary.wiley.com/doi/abs/10.1002/pssa. 2211020117

[34] J. M. Campillo Robles, E. Ogando, F. Plazaola, Positron lifetime calculation for the elements of the periodic table, Journal of Physics: Condensed Matter 19 (17) (2007) 176222. doi:10.1088/0953-8984/19/17/ 176222 .

URL https://doi .org/10.1088\%2F0953-8984\%2F19\%2F17\%2F176222

[35] P. Staikov, N. Djourelov, Simulations of $\langle 100\rangle$ edge and $1 / 2\langle 111\rangle$ screw dislocations in $\alpha$-iron and tungsten and positron lifetime calculations, Physica B: Condensed Matter 413 (2013) 59 - 63. doi:10.1016/j.physb.2012.12.026.

URL http://www.sciencedirect.com/science/article/pii/ S092145261201068X

[36] M. Eldrup, Positron studies of gases and gas bubbles in metals, Materials 
Science Forum 105 (1992) 229-248. doi:10.4028/www.scientific. net/MSF . 105-110.229.

[37] R. Steindl, G. Kögel, P. Sperr, P. Willutzki, D. Britton, W. Triftshäuser, Positron lifetimes on clean metallic surfaces, Materials Science Forum 105 (1992) 1455-1458. doi:10.4028/www.scientific.net/MSF. 105-110.1455.

[38] K. Sato, A. Hirosako, K. Ishibashi, Y. Miura, Q. Xu, M. Onoue, Y. Fukutoku, T. Onitsuka, M. Hatakeyama, S. Sunada, T. Yoshiie, Quantitative evaluation of hydrogen atoms trapped at single vacancies in tungsten using positron annihilation lifetime measurements: Experiments and theoretical calculations, Journal of Nuclear Materials 496 (2017) 9 -17. doi:10.1016/j.jnucmat.2017.09.002.

URL http://wWw.sciencedirect.com/science/article/pii/ S0022311517309303

[39] R. Krause-Rehberg, H. S. Leipner, Positron Annihilation in Semiconductors, Vol. 127, Springer-Verlag Berlin Heidelberg, 1999, Springer Series in Solid State Sciences.

[40] M. Zibrov, S. Ryabtsev, Y. Gasparyan, A. Pisarev, Experimental determination of the deuterium binding energy with vacancies in tungsten, Journal of Nuclear Materials 477 (2016) 292 - 297. doi:10.1016/j.jnucmat.2016.04.052.

URL http://www.sciencedirect.com/science/article/pii/ S002231151630174X

[41] A. van Veen, H. Filius, J. de Vries, K. Bijkerk, G. Rozing, D. Segers, Hydrogen exchange with voids in tungsten observed with TDS and PA, Journal of Nuclear Materials 155-157 (1988) 1113 - 1117. doi : 10.1016/0022-3115(88)90478-3.

URL http://www.sciencedirect.com/science/article/pii/ 0022311588904783

[42] S. Ryabtsev, Y. Gasparyan, M. Zibrov, A. Shubina, A. Pisarev, Deuterium thermal desorption from vacancy clusters in tungsten, Nuclear Instruments and Methods in Physics Research Section B 382 (2016) 101 - 104. doi:10.1016/j.nimb.2016.04.038. 
URL http://www.sciencedirect.com/science/article/pii/ S0168583X16301410

[43] A. van Veen, Thermal helium desorption spectrometry (THDS) as a tool for the study of vacancies and self-interstitials, Materials Science Forum 15 (1987) 3-24. doi:10.4028/www.scientific.net/MSF.15-18.3.

[44] D. R. Mason, D. Nguyen-Manh, C. S. Becquart, An empirical potential for simulating vacancy clusters in tungsten, Journal of Physics: Condensed Matter 29 (50) (2017) 505501.

URL http://stacks .iop.org/0953-8984/29/i=50/a=505501

[45] N. Castin, A. Bakaev, G. Bonny, A. Sand, L. Malerba, D. Terentyev, On the onset of void swelling in pure tungsten under neutron irradiation: An object kinetic monte carlo approach, Journal of Nuclear Materials 493 (2017) 280 - 293. doi:10.1016/j.jnucmat.2017.06.008.

URL http://www.sciencedirect.com/science/article/pii/ S0022311517301083

[46] X. Hu, T. Koyanagi, M. Fukuda, Y. Katoh, L. L. Snead, B. D. Wirth, Defect evolution in single crystalline tungsten following low temperature and low dose neutron irradiation, Journal of Nuclear Materials 470 (2016) 278 - 289. doi:10.1016/j.jnucmat. 2015.12.040. URL http://www.sciencedirect.com/science/article/pii/ S0022311515304098 